Research Paper

\title{
Thymoquinone inhibits metastasis of renal cell carcinoma cell 786-O-SI3 associating with downregulation of MMP-2 and $\mathrm{u}-\mathrm{PA}$ and suppression of PI3K/Src signaling
}

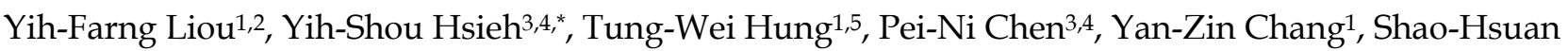 \\ $\mathrm{Kao}^{3}$, Shu-Wen Lin ${ }^{3}$, Horng-Rong Chang ${ }^{5,6} \bowtie$ \\ 1. Institute of Medicine, Chung Shan Medical University, Taichung, Taiwan \\ 2. Department of Internal Medicine, Feng Yuan Hospital, Ministry of Health and Welfare, Taiwan. \\ 3. Institute of Biochemistry, Microbiology and Immunology, Chung Shan Medical University, Taichung, Taiwan \\ 4. Clinical Laboratory, Chung Shan Medical University Hospital, Taichung, Taiwan \\ 5. Division of Nephrology, Department of Internal Medicine, Chung Shan Medical University Hospital, Taichung, Taiwan \\ 6. School of Medicine, Chung Shan Medical University, Taichung, Taiwan \\ ${ }^{*}$ Yih-Shou Hsieh contributed equally as first author. \\ $\triangle$ Corresponding author: Horng-Rong Chang MD, PhD, Division of Nephrology, Department of Medicine, Chung Shan Medical University Hospital, Taichung, \\ Taiwan. E-mail: chrcsmu@gmail.com, TEL: +886-4-24739595 ext. 34704 \\ (C) Ivyspring International Publisher. This is an open access article distributed under the terms of the Creative Commons Attribution (CC BY-NC) license \\ (https://creativecommons.org/licenses/by-nc/4.0/). See http://ivyspring.com/terms for full terms and conditions.
}

Received: 2019.01.02; Accepted: 2019.04.11; Published: 2019.05.10

\begin{abstract}
Phytochemicals represent an important source of novel anticancer and chemotherapeutic agents. Thymoquinone (TQ) is the major bioactive phytochemical derived from the seeds of Nigella sativa and has shown potent anticancer activities. In this study, we aimed to investigate the anticancer activity of Thymoquinone on the human renal carcinoma cell 786-O-SI3 and the underlying mechanism. By using cell proliferation assay, wound healing, and invasion assay, we found that Thymoquinone did not affect the viability of 786-O-SI3 and human kidney-2, but clearly inhibited the migration and invasion of 786-O-SI3. Further zymography and immunoblotting analysis showed that Thymoquinone downregulated the activity and expression of matrix metalloproteinase (MMP)-2 and urokinase-type plasminogen activator (u-PA) and attenuated the adhesion of 786-O-SI3 to type I and type IV collagen. Kinase cascade assay indicated that Thymoquinone inhibited the phosphorylation of phosphatidylinositol 3-kinase, Akt, Src, and Paxillin. In addition, Thymoquinone also decreased the level of fibronectin, N-cadherin, and Rho A. In parallel, Thymoquinone dose-dependently suppressed the transforming growth factor (TGF)- $\beta$-promoted u-PA activity and expression, as well as the cell motility and invasion of 786-O-SI3. Furthermore, tumor xenograft model revealed that Thymoquinone in vivo inhibited the 786-O-SI3 metastasizing to the lung. Collectively, these findings indicate that Thymoquinone inhibits the metastatic ability of 786-O-SI3, suggesting that Thymoquinone might be beneficial to promote the chemotherapy for renal cell carcinoma.
\end{abstract}

Key words: thymoquinone; renal carcinoma; invasion; matrix metalloproteinase; urokinase-type plasminogen activator

\section{Introduction}

Renal cell carcinoma (RCC) is the major type of human kidney cancer. Approximately 64,000 new RCC cases occurred during 2017 and cause 14,000 deaths in the USA [1]. Several risk factors associating with RCC has been proven, including hypertension, obesity, and smoking [2,3]. Among the patients with RCC, approximate $80-90 \%$ cases belong to the clear cell subtype that usually exhibits chemotherapy or radiotherapy resistance [4]. Although surgical resection is primary and widely used for RCC 
treatment, the effectiveness of surgery still highly depends on the stage and grade of the malignancy. About $25 \%$ of RCC patients are diagnosed as advanced stage with local invasive and metastatic RCC with a median survival time of 13 months [5]. However, the impact of chemotherapy on patients with advanced RCC is not yet satisfactory [6].

Thymoquinone (TQ), systemically named as 2-methyl-5-isopropyl-1,4-benzoquinone, is an important ingredient derived from the seeds of Nigella sativa and has been shown to have a variety of biological activities, including anti-inflammation, anti-oxidation, anti-bacteria, and anti-cancer [7, 8]. There is increasing evidence that Thymoquinone has potent anticancer activity against different types of malignancies, such as cervical cancer [9], prostate cancer [10], bladder cancer [11], and ovarian cancer [12]. However, whether Thymoquinone will reduce the motility and invasiveness of RCC cells has not been fully investigated.

Matrix metalloproteinases (MMPs) and serine proteases of the plasminogen activation system such as urokinase-type plasminogen activator (u-PA) play key roles in the disruption of extracellular matrix (ECM) during cancer metastasis [13, 14]. u-PA is an important serine protease involved in the degradation of ECM and is associated with cell division, adhesion, and migration [15]. In addition to involving the degradation of ECM, u-PA also converts proMMPs to active MMPs including MMP-2, a type IV collagenase belonging to MMP family, leading to proteolysis of ECM. The proteolysis cascade not only accelerates degradation of ECM but also promotes the invasion and metastasis of tumors [16, 17]. Thus, inhibition of MMPs and u-PA is considered to be an important target for anti-metastasis. In this study, we aimed to investigate the anti-metastatic effects of Thymoquinone on RCC cell line 786-O-SI3, a potent invasive xenograft-derived $786-\mathrm{O}$ cell and its underlying mechanism. In vitro cell migration and invasion, zymography, and western blot were performed for the determination of metastatic characteristics and ability. In vivo xenograft model was used to monitor the metastasis of RCC.

\section{Materials and methods}

\section{Materials, reagents, and antibodies}

Xenograft-derived 786-O cell line 786-O-SI3 was established as previously described [18]. The chemicals commonly used were purchased from Sigma-Aldrich (St. Louis, MO, USA), including Thymoquinone, Giemsa, 2-propanol, 3-(4,5-Dimethylthiazol-2-yl)-2,5-diphenyltetrazolium bromide (MTT), 1-butanol, dimethyl sulfoxide
(DMSO), phosphate-buffered saline (PBS), sodium chloride $(\mathrm{NaCl})$, sodium dodecyl sulfate (SDS), Tris- $\mathrm{HCl}$, and trypsin/EDTA. Transforming growth factor-beta1 (TGF- $\beta 1$ ) was purchased from R\&D Systems (Minneapolis, MN, USA).

\section{Cell culture and Thymoquinone treatment}

RCC cell line 786-O-SI3 and human kidney-2 (HK-2; a human proximal tubule epithelial cell line) cells were obtained from the Bioresource Collection and Research Center (Hsinchu, Taiwan). 786-O-SI3 cells were cultured in Roswell Park Memorial Institute (RPMI) 1640 medium (Gibco BRL, Grand Island, NY, USA) containing $10 \% \mathrm{v} / \mathrm{v}$ fetal calf serum (FBS, Hyclone, GE Healthcare), 2 mM L-glutamine, $100 \mathrm{mg} / \mathrm{mL}$ streptomycin and 100 units $/ \mathrm{mL}$ penicillin (Sigma). HK-2 cells were cultured in a 1:1 mixture of Dulbecco's modified Eagle's medium and Ham's F12 medium (Gibco-BRL) supplemented with $10 \% \mathrm{v} / \mathrm{v}$ FBS. The cell cultures were incubated at $37^{\circ} \mathrm{C}$ in a humidified atmosphere with $5 \% \mathrm{CO}_{2}$.

For Thymoquinone treatment, cells were grown to $80 \%$ confluency and then incubated with Thymoquinone at the indicated concentrations (5 - 20 $\mu \mathrm{M}$ ) for $24 \mathrm{~h}$. DMSO treatment (final concentration $0.1 \%$ ) was used as sham control. The treated cells were harvested and washed with PBS for the subsequent analyses.

\section{Cell viability assessment using MTT cell proliferation assay}

Cell viability was determined by using an MTT colorimetric method as previously described [19]. Briefly, cells were seeded in 24-well plates at a density of $3 \times 10^{4}$ cells/well, treated with serial concentrations of Thymoquinone $(0-20 \mu \mathrm{M})$ at $37^{\circ} \mathrm{C}$ for $24 \mathrm{~h}$. Otherwise, cells were pretreated with Thymoquinone $(0-20 \mu \mathrm{M})$ for $2 \mathrm{~h}$ followed by incubated with or without $10 \mathrm{ng} / \mathrm{mL}$ TGF- $\beta 1$ for an additional $48 \mathrm{~h}$. Cell were washed with PBS, and then incubated with MTT solution $(5 \mathrm{mg} / \mathrm{mL})$ for $4 \mathrm{~h}$. The generation of formazan was solubilized with 2-propanol and analyzed by a Hitachi U-1900 spectrophotometer (Hitachi, Tokyo, Japan) at $563 \mathrm{~nm}$. The viable cell number was directly proportional to formazan production.

\section{Migration assay using wound healing}

Cells were incubated until reaching the confluent monolayer, and then the wounds were introduced by using culture-inserts (Ibidi $\mathrm{GmbH}$, Martinsried, Germany) to create a cleared line. After replacing with RPMI 1640 medium containing 1\% FBS and the indicated concentration of Thymoquinone, the cells were incubated at $37^{\circ} \mathrm{C}$ for $24 \mathrm{~h}$. The cells migrated 
into the wound area was photographed and counted at the 0,6 and $24 \mathrm{~h}$ by a microscope (CKX41: Olympus, Tokyo, Japan).

\section{Transmigration and invasion assessment}

786-O-SI3 cells were treated with Thymoquinone at the indicated concentrations for $24 \mathrm{~h}$. Otherwise, cells were pretreated with Thymoquinone $(0-20 \mu \mathrm{M})$ for $2 \mathrm{~h}$ followed by incubated with or without 10 $\mathrm{ng} / \mathrm{mL}$ TGF- $\beta 1$ for an additional $48 \mathrm{~h}$. After treatment, the cells were harvested and seeded in a Boyden chamber (Neuro Probe, Cabin John, MD, USA) at a cell density of $10^{4}$ cells/well and then incubated in serum-free medium at $37^{\circ} \mathrm{C}$ for $12 \mathrm{~h}$. For the invasion assessment, $10 \mu \mathrm{L}$ Matrigel ${ }^{\circledR}$ (BD Biosciences, Bedford, MA, USA) was applied into the membrane filters (pore size $8 \mu \mathrm{m}$, Neuro Probe, Cabin John, MD, USA) and the standard medium was added into the bottom chamber of the apparatus. After $24 \mathrm{~h}$ incubation, the filters were dried in a laminar flow hood, then the invaded cells were fixed with methanol and stained with Giemsa. Cell numbers were counted using a light microscope (CKX41; Olympus). The transmigration assessment was performed as described in the invasion assay except for use of Matrigel [20].

\section{Enzymatic activity assessment of MMP-2 and u-PA by zymography}

786-O-SI3 cells were treated with Thymoquinone at the indicated concentrations for $24 \mathrm{~h}$. Otherwise, cells were pretreated with Thymoquinone $(0-20 \mu \mathrm{M})$ for $2 \mathrm{~h}$ followed by incubated with or without 10 $\mathrm{ng} / \mathrm{mL}$ TGF- $\beta 1$ for an additional $24 \mathrm{~h}$. The activities of MMP-9 and u-PA in the cultured medium were determined by using gelatin-zymogram protease assays as previously described [21]. Briefly, the collected medium samples were reacted with the analysis buffer $(0.01 \%$ SDS, $50 \mathrm{mM}$ Tris- $\mathrm{HCl}, \mathrm{pH} 6.8)$ in the room temperature for $30 \mathrm{~min}$, loaded into the $8 \%$ SDS-gel containing $0.1 \%$ gelatin, and then electrophoresed at $150 \mathrm{~V}$ in an OWL P-1 apparatus (Alpha Multiservices, Inc., Conroe, TX, USA) for $3 \mathrm{~h}$. After the electrophoresis, the gels were washed with the $2 \%$ Triton X-100 in distilled water with gentle shaking at room temperature for $30 \mathrm{~min}$. Then, the washed gels were incubated with the reaction buffer (40 mM Tris- $\mathrm{HCl}, \mathrm{pH} 8.0$, containing $10 \mathrm{mM} \mathrm{CaCl} 2$ and $0.02 \% \mathrm{NaN}_{3}$ ) at $37^{\circ} \mathrm{C}$ for $12 \mathrm{~h}$, and stained with Coomassie brilliant blue R-250. u-PA activity was determined using the same method for MMP-2, and $0.1 \%$ gelatin was replaced with $2 \%$ casein and 20 $\mathrm{mg} / \mathrm{mL}$ plasminogen (Sigma). Electrophoresis and zymography were then performed for gelatin zymography.

\section{Cell adhesion assay}

Cell adhesion assay was performed as previously described [22]. Briefly, cells were seeded into 12-well plates coated with type I or type IV collagen at the density of $5 \times 10^{5}$ cells $/ \mathrm{mL}$ and incubated with the medium containing $10 \%$ FBS. The number of cells attached to the collagen was assessed on the first day after inoculation.

\section{Immunofluorescence assay}

The cells were pretreated with Thymoquinone for $2 \mathrm{~h}$ prior to stimulation with TGF- $\beta 1(10 \mathrm{ng} / \mathrm{mL})$ for $48 \mathrm{~h}$. Cells were grown on glass coverslips and fixed with $4 \%$ paraformaldehyde (Sigma) at room temperature for $12 \mathrm{~min}$. After washing with PBS, the fixed cells were blocked with $4 \%$ bovine serum albumin (Sigma) in PBS and then permeabilized with $0.1 \%$ Triton X-100 in PBS at room temperature for 90 min. Filamentous actin was detected by incubating the treated cells with rhodamine-conjugated phalloidin $(1: 200)$ at $4^{\circ} \mathrm{C}$ for $16 \mathrm{~h}$. The detection of nuclei was by counterstaining the cells with 4',6-diamidino-2-phenylindole (DAPI) at room temperature for $1 \mathrm{~h}$. The images were detected and photographed using a ZEISS Axioskop2 upright fluorescence microscope (Carl Zeiss AG, Oberkochen, Germany).

\section{Western blot}

Crude proteins extracted from whole cell lysates were separated by $12.5 \%$ SDS- polyacrylamide gel electrophoresis and transferred onto a nitrocellulose membrane (GE Healthcare) as previously described [23]. The transferred membrane was blocked with 5\% skimmed milk, incubated with primary antibodies, reacted with secondary antibodies, and then incubated with chemoluminescent reagent (Enhanced Chemiluminescence Plus detection kit, Amersham Life Sciences, Inc., Piscataway, NJ, USA).) for signal development. The chemiluminescent signals were acquired and quantitated using a Luminescent Image Analyzer LAS-4000 mini (GE Healthcare).

\section{Assessment of 786-O-SI3 metastasizing to lung using xenograft model}

Five-week-old male C57BL/6 mice were obtained from National Taiwan University Animal Center (Taipei, Taiwan) and maintained with a regular 12-h light/dark cycle and ad libitum access to a standard rodent diet (Laboratory Rodent Diet 5001; LabDiet, St. Louis, MO). 786-O-SI3 cells $\left(1 \times 10^{6}\right)$ were suspended in $0.1 \mathrm{~mL}$ PBS and then administrated into the mice via tail vein injection (Day-0). On the next day (Day-1), the treated mice were randomly divided into three groups ( $\mathrm{n}=5$ for each group) and daily fed 
by oral gavage with olive oil (Sham control) or Thymoquinone (10 and $20 \mathrm{mg} / \mathrm{kg}$ of body weight). Three untreated mice were used as wild-type controls. Tumor metastasis was monitored on the basis of luciferase activity in 786-O-SI3 cells; the photons emitted from the target site penetrated the mammalian tissue; these photons could be externally detected and quantified using a sensitive light imaging system. The treated mice were sacrificed using $\mathrm{CO}_{2}$ on the Day-42, the lungs were isolated and weighed and the metastasized nodules on the surface of the lungs were counted using a microscope (Axioskop 2 Plus, Carl Zeiss, Inc., Oberkochen, Germany). The lung samples were fixed in neutral buffered 5\% formalin (Sigma) and embedded in paraffin as described [24]. Sections were cut at a thickness of 3-5 $\mu \mathrm{m}$ and stained with hematoxylin and eosin. The histopathological changes, including cell morphology and metastatic tumor cells, were examined by light microscopy.

\section{Statistical analysis}

Statistical significance was examined by using Student's t-test (SigmaStat 2.0; Jandel Scientific, San Rafael, CA). $P$ value less than 0.05 was considered as statistically significant difference.

\section{Results}

\section{Thymoquinone did not affect the cell viability of 786-O-SI3}

The chemical structure was presented in Fig. 1A. We first investigated the effects of Thymoquinone on cell viability of 786-O-SI3, a previously established xenograft-derived 786-O cell with highly invasiveness [18]. As shown in Fig. 1B, although 24 h-treatments of Thymoquinone at $20 \mu \mathrm{M}$ slightly decreased the cell viability of $786-\mathrm{O}-\mathrm{SI} 3$ to $93.4 \pm 2.5 \%$ of control, no statistical significance was observed between the treatments and control $(P>0.05)$. In addition, effects of Thymoquinone on cell viability of non-tumorigenic kidney cell HK-2 was also explored, and the results showed that Thymoquinone insignificant influenced the cell viability of $\mathrm{HK}-2$ cell (Fig. 1C, P>0.05). Collectively, these findings revealed that Thymoquinone had no significant cytotoxicity against highly aggressive RCC cells 786-O-SI3 and non-malignant renal cells HK-2.

\section{Thymoquinone reduced the invasion and cell motility of 786-O-SI3}

As Thymoquinone showed no significant cytotoxicity to the renal cells, we next investigate whether Thymoquinone influenced the invasion and cell motility of 786-O-SI3. As shown in Fig. 2A, the invasion assay exhibited that Thymoquinone significantly reduced the invasive ability of 786-O-SI3 cells. Similar to the invasion assay, the transmigration analysis revealed that Thymoquinone significantly decreased the number of transmigrated cells in a dose-dependent manner (Fig. 2B, P<0.001). In addition, the wound healing assay also showed that Thymoquinone treatment clearly inhibited the migration of 786-O-SI3 cell as compared to the control (Fig. 2C, $P<0.001$ ). Taken together, these observations indicated that Thymoquinone was able to inhibit the cell migration and invasion of 786-O-SI3.

A<smiles>CC1=CC(=O)C(C(C)C)=CC1=O</smiles>

Thymoquinone

B 786-O SI3

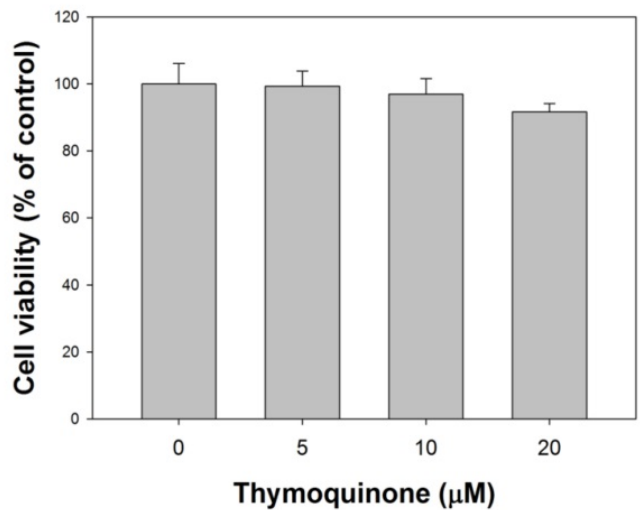

C

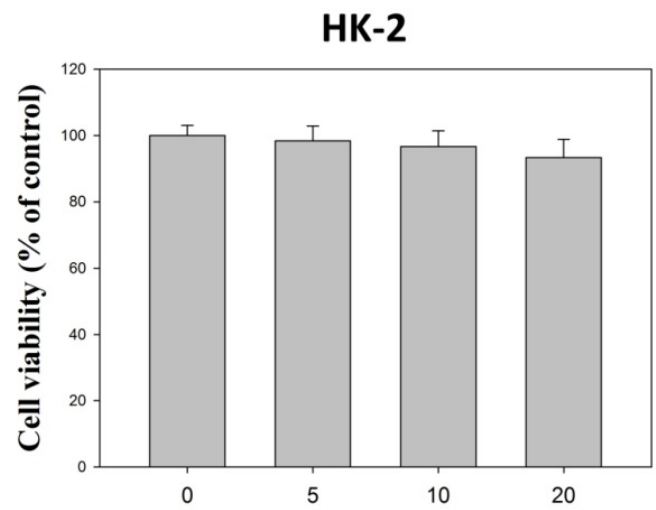

Thymoquinone $(\mu \mathbf{M})$

Figure 1. Effects of Thymoquinone on the cell viability of 786-O-SI3. A, The chemical structure of Thymoquinone. B and C, Cells were treated with Thymoquinone for $24 \mathrm{~h}$, and then the cell viability was performed using MTT assay. The cell viability was presented as percentage of control. No statistical significance was observed between the Thymoquinone treatments and control. 
A
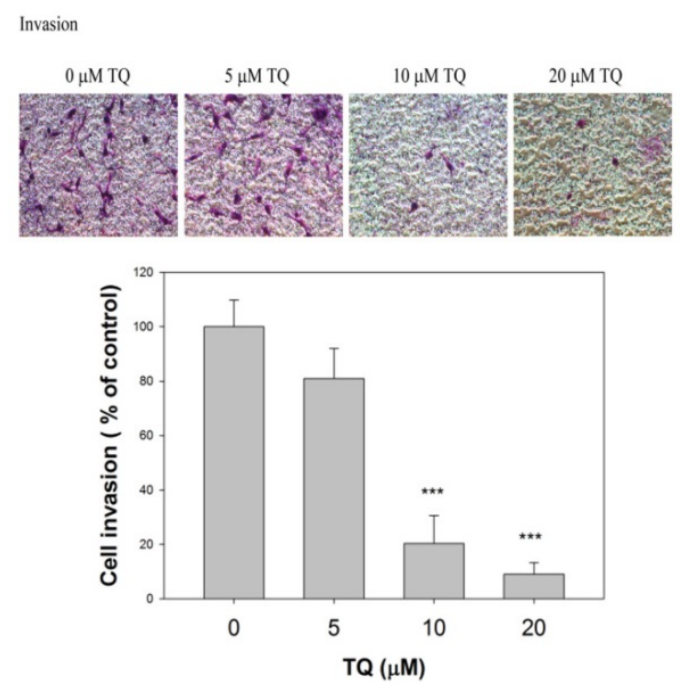

C

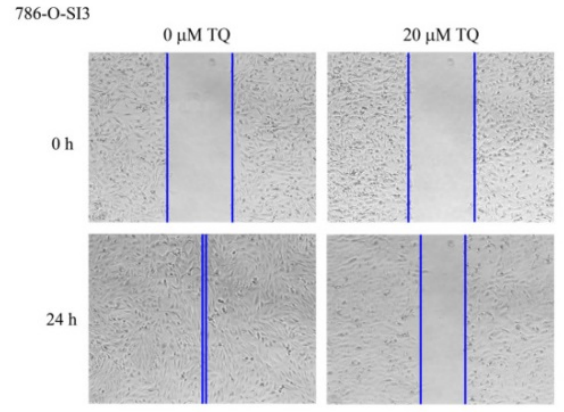

\section{B}
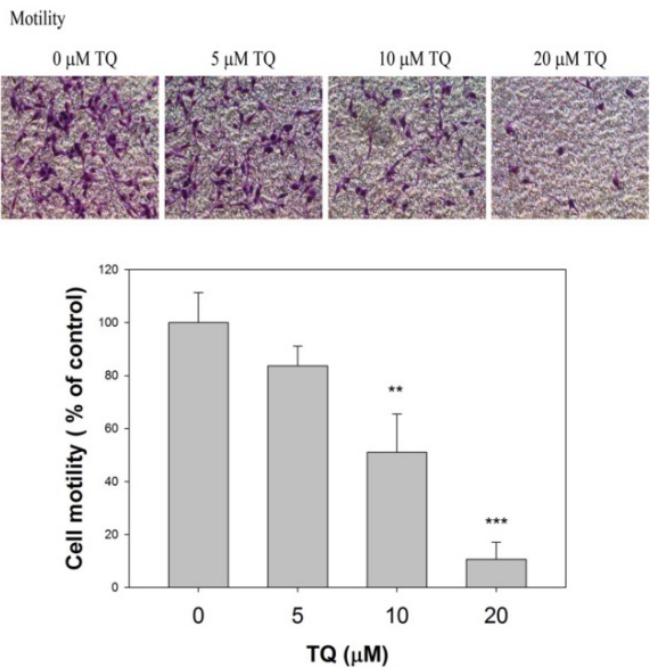

786-O-SI3

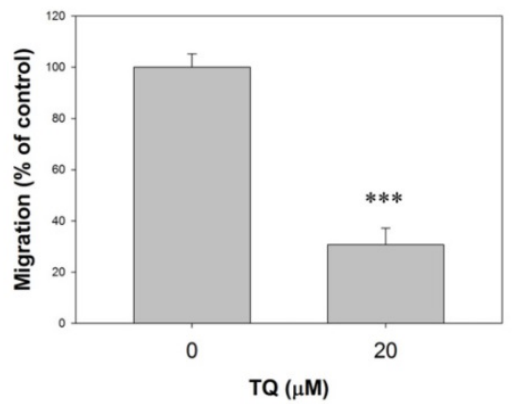

Figure 2. Thymoquinone reduced the invasion and cell motility of 786-O-SI3. Cells were treated with Thymoquinone at the indicated concentrations for $24 \mathrm{~h}$, and then subjected to invasion assay $(A)$, transmigration assay $(B)$, and wound healing migration assay $(C)$. The quantitation of invaded cells and migrated cells was presented as percentage of control. ** and $* * *, P<0.01$ and 0.001 as compared to control.

\section{Thymoquinone inhibited the expression and secretion of MMP-2 and u-PA by 786-O-SI3}

Our previous study has shown that 786-O-SI3 is highly invasive due to the high expression of MMP-2 and u-PA [18]. Thus, we further examined whether Thymoquinone inhibited the secretion and protein and gene expression of these proteases. As shown in Fig. $3 \mathrm{~A}$ and $3 \mathrm{~B}$, the zymography experiments revealed that Thymoquinone dose-dependently decreased the proteolytic activity of secreted MMP-2 and u-PA $(P<0.001)$. In parallel, we also found that Thymoquinone eminently inhibited the protein expression of MMP-2 and u-PA (Fig. 3C and 3D). Furthermore, we tested whether Thymoquinone suppressed the gene transcription of MMP-2 and u-PA by using reporter assay, and the results showed that Thymoquinone significantly suppressed the gene transcription of both MMP-2 and u-PA (Fig. 3E and $3 F, P<0.01)$. Taken together, these findings indicated that Thymoquinone was able to inhibit the transcription of MMP-2 and u-PA, resulting in a subsequent decrease in protein expression and secretion.

\section{Thymoquinone attenuated the cell adhesion of 786-O-SI3 to type I and type IV collagen}

The adhesion strength was observed to be different in multiple cancer cell lines, and thus the adhesion strength was presumed to be a general marker for metastatic cells [25]. Accordingly, the adhesive strength of 786-O-SI3 to collagens was examined. As shown in Fig. 4A, $20 \mu \mathrm{M}$ Thymoquinone significantly decreased the number of cells adhered to type I collagen to $80.46 \pm 9.0 \%$ of control $(P<0.05)$. In parallel, $20 \mu \mathrm{M}$ Thymoquinone also decreased the number of cells adhered to type IV collagen to $71.59 \pm 6.3 \%$ of control (Fig. $4 \mathrm{~B}, P<0.001$ ). These observations showed that Thymoquinone inhibited the adhesion of 786-O-SI3 to type I and type IV collagen. 
A
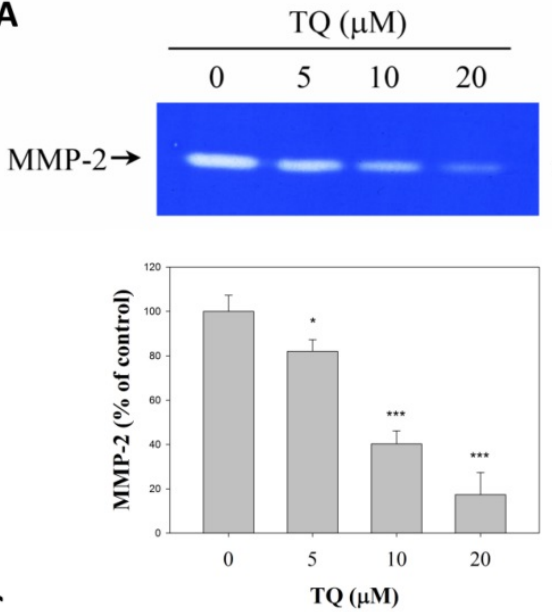

C

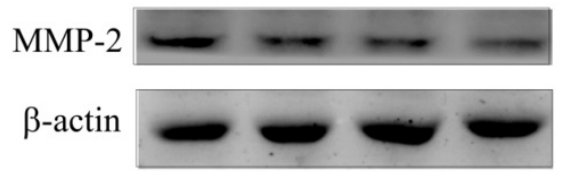

E

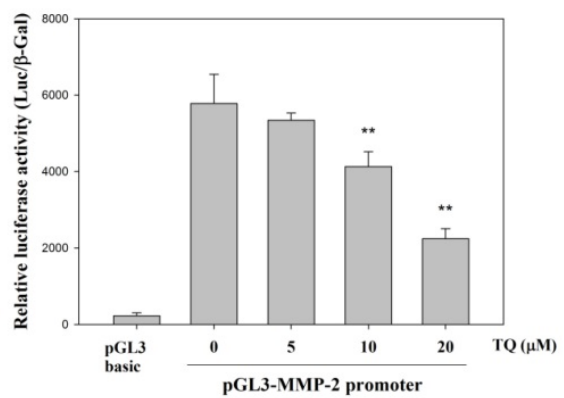

B
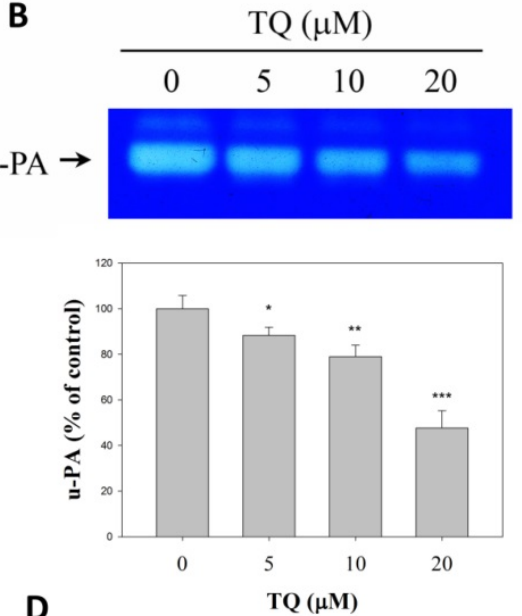

D

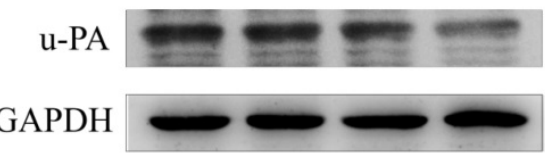

$\mathbf{F}$

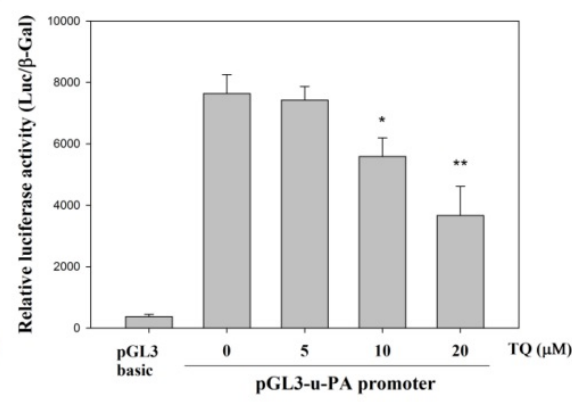

Figure 3. Thymoquinone inhibited the expression and secretion of MMP-2 and u-PA by 786-O-SI3. Cells were treated with Thymoquinone for 24 h, then the supernatants were collected for MMP-2 zymography assay $(A)$ and $u$-PA zymography assay (B), and the treated cells were harvested, lysed, and subjected to western blot for determining the levels of MMP-2 and u-PA. *, ** and $* * *, P<0.05,0.01$ and 0.001 as compared to control.

Thymoquinone suppressed the phosphoinositide 3-kinases (PI3K)/Akt and Src/Paxillin signaling cascade in 786-O-SI3 cells contributing to the downregulation of MMP-2 and the attenuation of invasion

Considering that Thymoquinone inhibited the invasion and migration and downregulated the transcription of MMP-2 and u-PA in 786-O-SI3 cells, the effects of Thymoquinone on PI3K/Akt and $\mathrm{Src} /$ Paxillin cascade that mediated the expression of MMP-2 and u-PA were further explored. The western blot results showed that Thymoquinone treatments decreased PI3K level and Akt phosphorylation (Fig. 5A), attenuated the phosphorylation of Src and Paxillin (Fig. 5B), and downregulated the protein level of fibronectin, N-cadherin, and Rho A (Fig. 5C). Taken together, these findings suggested that Thymoquinone suppressed the PI3K/Akt and Src/Paxillin signaling-mediated expression of cell adhesion molecules and MMP-2 and subsequent inhibition of invasion.
Thymoquinone antagonized the TGF- $\beta 1$-promoted cell motility, invasion, and cytoskeleton remodeling of 786-O-SI3 cells

Typical TGF- $\beta 1$ signaling can promote invasion and metastasis of cancer cell leading to tumor progression in the late stages [26]. Therefore, whether Thymoquinone antagonized the TGF- $\beta 1$-promoting cell motility and invasion were subsequently investigated. As shown in Fig. 6A, 10 ng/mL TGF- $\beta 1$ and the combination of $10 \mathrm{ng} / \mathrm{mL}$ TGF- $\beta 1$ and Thymoquinone $(5-20 \mu \mathrm{M})$ did not influence the cell viability of $786-\mathrm{O}-\mathrm{SI} 3 \quad(P>0.05)$. By using gelatin zymography protease assay, we observed that TGF- $\beta 1$ treatment significantly increased the activity of secreted u-PA to $187.3 \pm 11.0 \%$ of the control $(P<$ 0.05 ), and the increase in u-PA activity was reduced in a dose-dependent manner by the combined treatment with Thymoquinone (Fig. 6B). Although TGF- $\beta 1$ treatment did not promote invasion of 786-O-SI3 cells, Thymoquinone was able to inhibit the invasion of 
$786-\mathrm{O}-\mathrm{SI} 3$ cells in the presence of TGF- $\beta 1 \quad(P<0.001$, Fig. 6C). Similarly, TGF- $\beta 1$ treatment significantly increased the cell motility of 786-O-SI3 cells to $128.4 \pm$ $10.5 \%$ of the control $(P<0.05)$, and the increase cell motility was reduced in a dose-dependent manner by the combined treatment with Thymoquinone (Fig. 6D). Moreover, we also explored the effect of Thymoquinone on TGF- $\beta 1$-induced cytoskeletal changes involving the promotion of cell movement. As shown in Fig. 6E, TGF- $\beta 1$ treatment induced cytoskeletal rearrangement in 786-O-SI3 cells and contributed to morphological changes from epithelial to mesenchymal phenotype, and the cytoskeletal rearrangement and morphological changes were restored by the combined treatment with Thymoquinone. Taken together, these findings showed that Thymoquinone inhibited the TGF- $\beta 1$-promoted invasion and cell migration of 786-O-SI3 cells, which may attribute to the reduction of $\mathrm{u}-\mathrm{PA}$ production and suppression of cytoskeletal rearrangement.

A

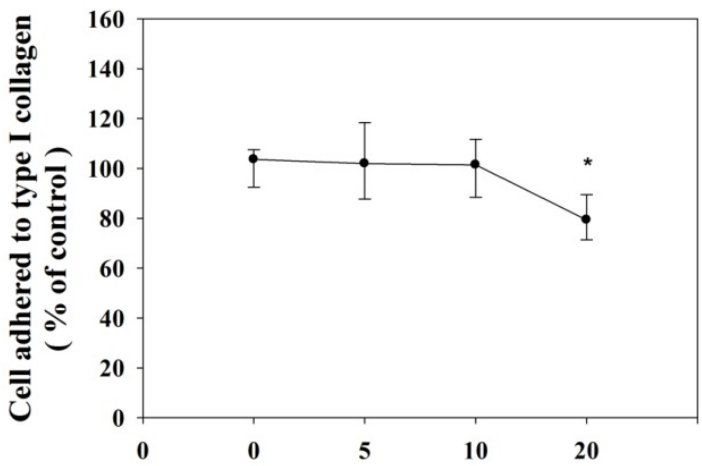

B

Thymoquinone $(\mu \mathrm{M})$

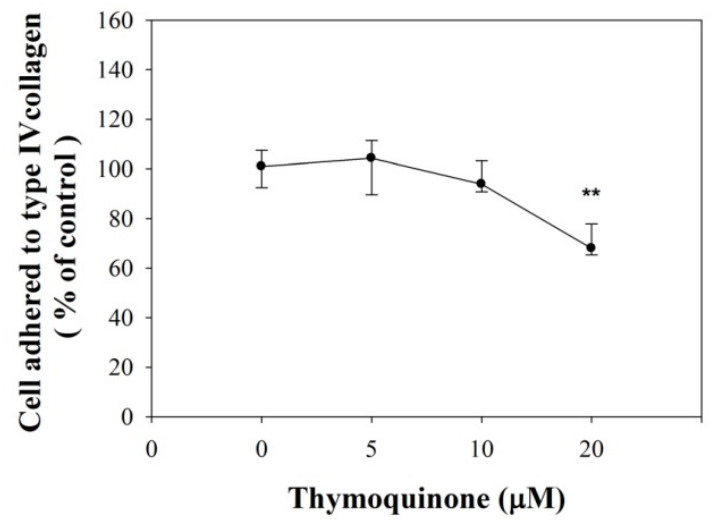

Figure 4. Thymoquinone attenuated the cell adhesion of 786-O-SI3 to type I and type IV collagen. Cells were incubated on (A) type I or (B) type II collagen-coated plates and treated with Thymoquinone at serial concentrations for $24 \mathrm{~h}$, then wash the detached cells and the adhered cells were counted. The cell adhesion was presented as the percentage of adhered cells to the control.* and $* *, P<0.05$ and 0.01 as compared to control.

\section{Thymoquinone in vivo inhibited the transfer of 786-O-SI3 cells to the lungs}

We next investigated whether Thymoquinone inhibited the metastasis of 786-O-SI3 cells to the lung by using xenograft mouse model. As shown in Fig. 7A, Thymoquinone administration significantly reduced the number of 786-O-SI3 cells transferred to the lung in live mice $(P<0.001)$. In addition, Thymoquinone decreased the lung weights of xenografted mice (Fig. 7B). The histological examination also showed that Thymoquinone administration attenuated the colonies of 786-O-SI3 cells in lung tissues (Fig. 7C). Collectively, these results revealed that Thymoquinone in vivo inhibited the lung metastasis of 786-O-SI3 cells.

\section{A}
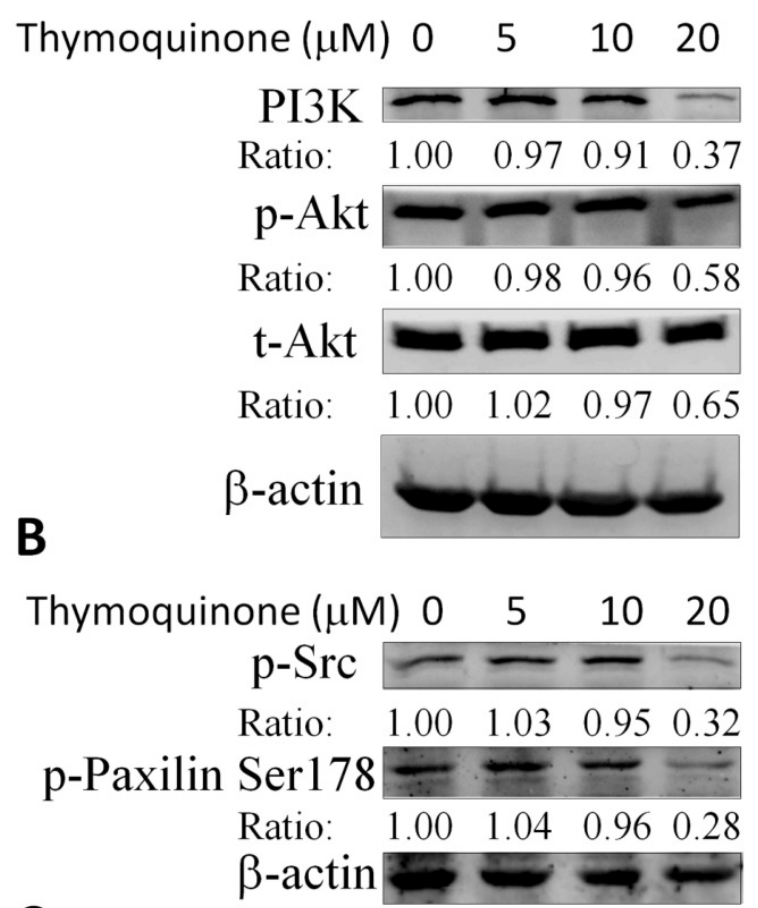

C

Thymoquinone $(\mu \mathrm{M}) \quad 0 \quad 5 \quad 10 \quad 20$ Fibronectin - - Ratio: $\quad \begin{array}{llll}1.00 & 0.95 & 0.95 & 0.36\end{array}$ N-cadherin

\begin{tabular}{|c|c|c|c|c|}
\hline Ratio: & 1.00 & 0.99 & 0.92 & 0. \\
\hline RhoA & - & $\longrightarrow$ & $\omega$ & $=$ \\
\hline Ratio: & 1.00 & 1.01 & 0.97 & \\
\hline B-actin & & & & \\
\hline
\end{tabular}

Figure 5. Thymoquinone suppressed the PI3K/Akt and Src/Paxillin signaling cascade in 786-O-SI3 cells contributing to the downregulation of MMP-2 and the attenuation of invasion. Cells were treated with Thymoquinone at the indicated concentrations for $24 \mathrm{~h}$, then harvested the cells for cell lysis and the subsequent western blot $(\mathrm{A}-\mathrm{C})$. A result representing three separate experiments is shown. 


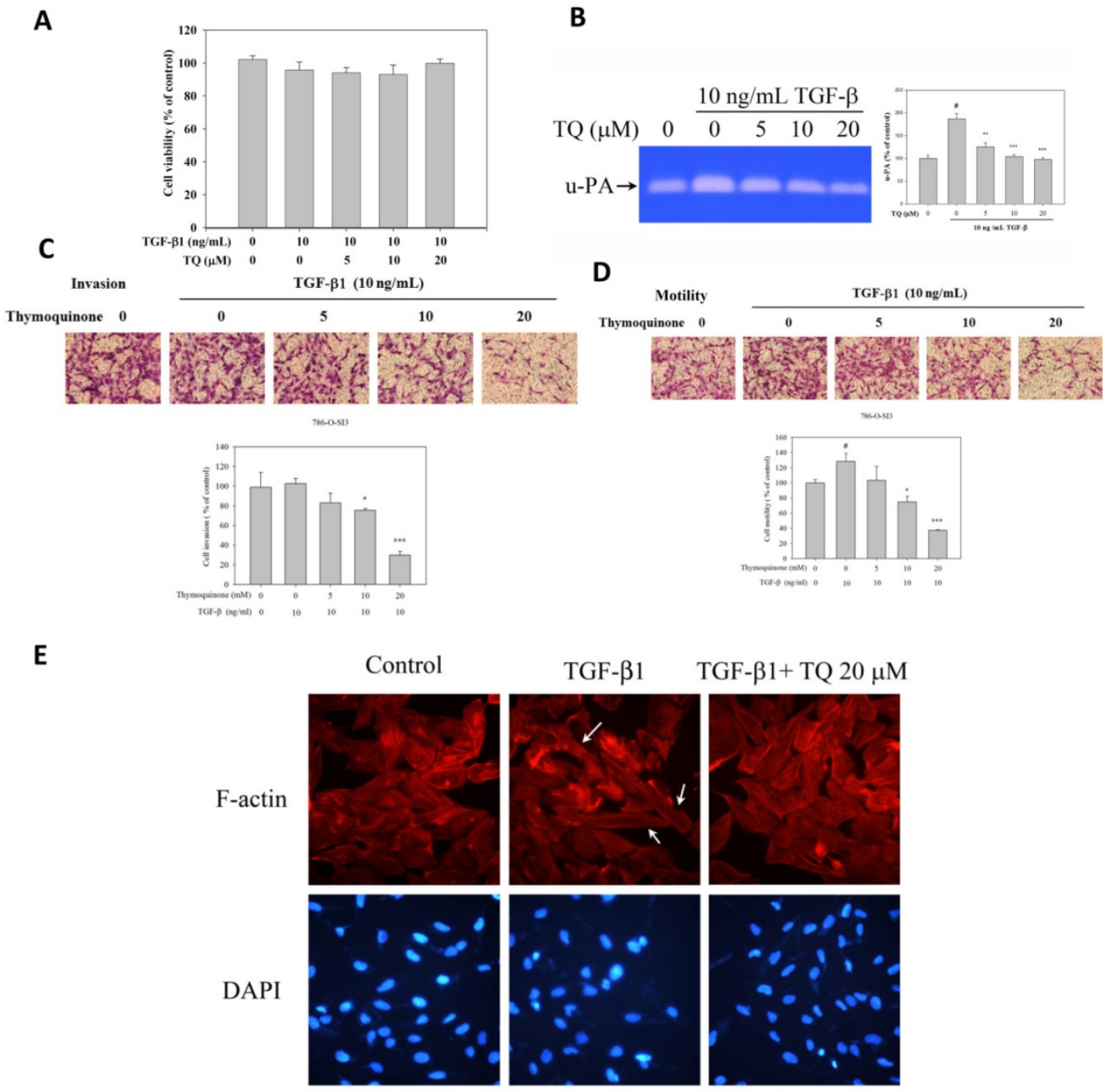

Figure 6. Thymoquinone antagonized the TGF- $\beta 1$-promoted cell motility, invasion, and cytoskeleton remodeling of 786-O-SI3 cells. Cells were treated with Thymoquinone or Thymoquinone plus TGF- $\beta 1$ for 24 or $48 \mathrm{~h}$, then the cells were subjected to (A) cell viability assay, and the cultured medium was collected for u-PA activity assay (B). Cells were cultured on Boyden chamber coated with Matrigel or not, treated with TGF- $\beta 1$ (10 ng/mL) or TGF- $\beta 1$ (10 ng/mL) combining with Thymoquinone (5-20 $\mu \mathrm{M})$ for $24 \mathrm{~h}$, and then analyzed by using invasion assay (C), transmigration assay (D), or immunofluorescence detection of F-actin and DAPI (E). (\#, $P<0.05$ compared with control; **, $P<0.01 ; * * *, P<0.001$ compared with TGF- $\beta 1$-treated group).

\section{Discussion}

In the present study, we demonstrate the anti-metastatic activity of Thymoquinone on highly invasive RCC cell line 786-O-SI3, which may attribute to inhibition of MMP-2 and u-PA, suppression of PI3K/Akt and Src/Paxillin signaling, and the cytoskeletal changes. The extracellular-signal-regulated kinase and Src signaling have been reported to involve in the regulation of u-PA and MMPs expression and the modulation of motility and invasion of lung cancer cell [27]. In addition, Akt signaling is also reported to modulate MMP-2 and regulate actin organization [28]. Our results showed that Thymoquinone not only inhibits the PI3K/Akt activation involving in MMP-2 expression but also suppresses the Src/Paxillin axis associating with cell adhesion and actin organization, indicating that Thymoquinone possesses potent anti-metastatic activity on RCC cells.

The cytoskeleton is a highly dynamic network of actin polymers and a variety of related proteins that mediate various important biological functions in eukaryotic cells, including extracellular movement and structural support. Therefore, the organization of the actin cytoskeleton is closely regulated in time and space. Disrupting normal regulation of actin cytoskeleton and its regulatory proteins Rho guanosine triphosphatases (GTPase) may result in carcinogenic transformation and cancer progression $[29,30]$. In this study, our findings reveal that Thymoquinone inhibits phosphorylation of Src and paxillin, as well as decreases level of fibronectin, $\mathrm{N}$-cadherin, and Rho A. It suggests that Thymoquinone can regulate actin cytoskeleton and cell adhesion molecules through modulation of these cytoskeletal regulatory proteins, and affect cytoskeleton and cell motility. 
A
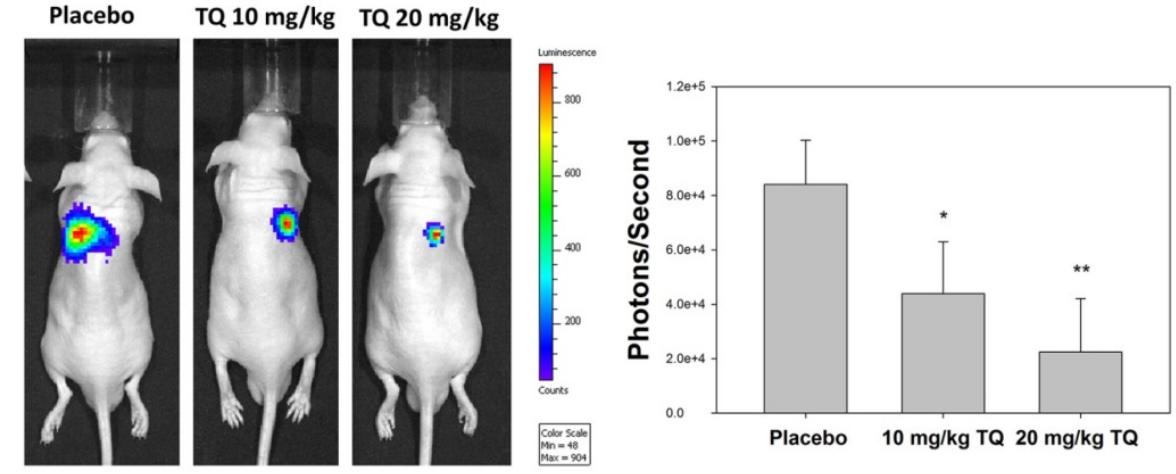

B
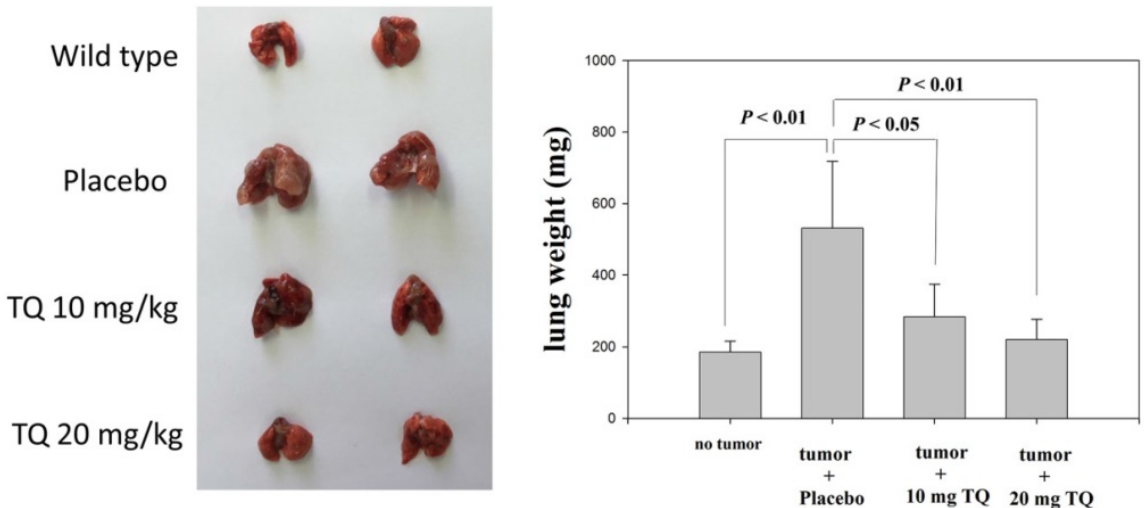

C

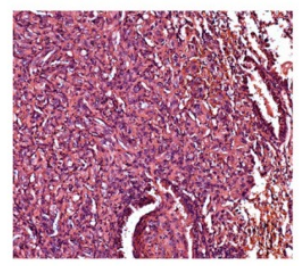

786-O SI3

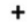

Placebo

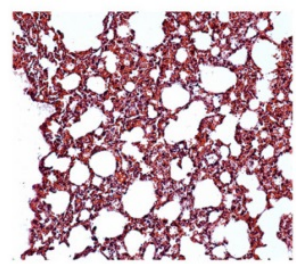

Wild type
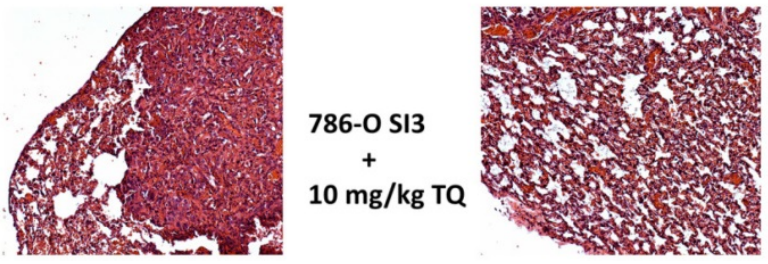

786-O SI3

$20 \mathrm{mg} / \mathrm{kg}$ TQ

Figure 7. Thymoquinone inhibited the lung metastasis of 786-O-SI3 cells in xenograft mouse model. Mice receiving 786-O-SI3 cells transfected with luciferase-expressing vector were orally administrated with Thymoquinone $10 \mathrm{mg} / \mathrm{kg}$ or $20 \mathrm{mg} / \mathrm{kg}$ per day. After 42 consecutive day administration, luciferase substrate was injected via intraperitoneal and the chemiluminescent images were obtained and quantitated by using IVIS imaging system (A), and then the lung samples were acquired from the sacrificed mice for the determination of lung weight (B) and for histological analysis using HE staining (C). Oral administration of olive oil was used as placebo.

TGF- $\beta 1$ signaling has been demonstrated to promote tumor progression by inducing epithelial to mesenchymal transition and enhancing invasion and metastasis [31]. In the present study, we observe that TGF- $\beta 1$ does not affect cell viability and invasion but significantly enhances the motility of 786-O-SI3 cells and actin reorganization, and Thymoquinone can inhibit the invasion, enhanced motility, and actin reorganization of 786-O-SI3 cells in response to TGF- 31 . In addition, Thymoquinone also in vivo inhibits the transfer of 786-O-SI3 cells to lungs in xenografted mouse model. These findings indicate that TGF- $\beta 1$ signaling may promote the metastasis of RCC and suggest that Thymoquinone might antagonize the TGF- $\beta 1$ signaling to inhibit the metastasis of RCC. However, the effects of Thymoquinone on TGF- $\beta 1$ signaling in RCC need further investigation.

MMP-2, MMP-9 and u-PA play central roles in RCC metastasis and involve in promoting the invasion, migration, and angiogenesis during RCC progression $[32,33]$. Similarly, our observations show that MMP-2 and u-PA are highly produced by the highly invasive RCC cell 786-O-SI3. In addition, our 
findings also demonstrate that TGF- $\beta 1$ treatment can induce more u-PA production by 786-O-SI3 cells. Notably, our results reveal that Thymoquinone can suppress the expression and production of MMP-2 and $\mathrm{u}-\mathrm{PA}$ in 786-O-SI3 cells in the presence or absence of TGF- $\beta 1$. These findings reveal that TGF- $\beta 1$ dose not significantly antagonize the inhibitory expression and production of u-PA by Thymoquinone, suggesting that Thymoquinone can still exert its anti-metastatic activity on 786-O-SI3 cells in the high TGF- $\beta 1$ microenvironment during tumor progression.

In conclusion, this study elucidated the anti-metastatic mechanism of Thymoquinone, which is mainly due to the inhibition of invasion, cell movement, expression, and production of MMP-2 and $\mathrm{u}-\mathrm{PA}$, cell adhesion and cytoskeletal reorganization. These findings not only demonstrate the effective anti-metastatic activity of Thymoquinone, but also provide the potential to use Thymoquinone as a therapeutic supplement for RCC treatment.

\section{Acknowledgments}

This study was financially supported by clinical research grants from the Ministry of Science and Technology, Taiwan [105-2314-B-040-014-MY3 and 106-2320-B-040-020-MY3].

\section{Competing Interests}

The authors have declared that no competing interest exists.

\section{References}

1. Siegel RL, Miller KD, Jemal A. Cancer Statistics, 2017. CA Cancer J Clin. 2017;67:7-30.

2. Hunt JD, van der Hel OL, McMillan GP, et al. Renal cell carcinoma in relation to cigarette smoking: meta-analysis of 24 studies. Int J Cancer. 2005;114:101-8.

3. Chow WH, Gridley G, Fraumeni JF, et al. Obesity, hypertension, and the risk of kidney cancer in men. N Engl J Med. 2000;343:1305-11.

4. Torre LA, Bray F, Siegel RL, et al. Global cancer statistics, 2012. CA Cancer J Clin. 2015;65:87-108.

5. Al Otaibi M, Abou Youssif T, Alkhaldi A, et al. Renal cell carcinoma with inferior vena caval extention: impact of tumour extent on surgical outcome. BJU Int. 2009;104:1467-70.

6. Amato RJ. Chemotherapy for renal cell carcinoma. Semin Oncol. 2000;27:177-86.

7. Ravindran J, Nair HB, Sung B, et al. Thymoquinone poly (lactide-co-glycolide) nanoparticles exhibit enhanced anti-proliferative, anti-inflammatory, and chemosensitization potential. Biochem Pharmacol. 2010;79:1640-7.

8. Inci $\mathrm{M}$, Davarci $\mathrm{M}$, Inci $\mathrm{M}$, et al. Anti-inflammatory and antioxidant activity of thymoquinone in a rat model of acute bacterial prostatitis. Hum Exp Toxicol. 2013;32:354-61.

9. Li J, Khan MA, Wei C, et al. Thymoquinone Inhibits the Migration and Invasive Characteristics of Cervical Cancer Cells SiHa and CaSki In Vitro by Targeting Epithelial to Mesenchymal Transition Associated Transcription Factors Twist1 and Zeb1. Molecules. 2017;22.

10. Kou B, Liu W, Zhao W, et al. Thymoquinone inhibits epithelial-mesenchymal transition in prostate cancer cells by negatively regulating the TGF-beta/Smad2/3 signaling pathway. Oncol Rep. 2017;38:3592-8.

11. Iskender B, Izgi K, Hizar E, et al. Inhibition of epithelial-mesenchymal transition in bladder cancer cells via modulation of mTOR signalling. Tumour Biol. 2016;37:8281-91.

12. Liu X, Dong J, Cai W, et al. The Effect of Thymoquinone on Apoptosis of SK-OV-3 Ovarian Cancer Cell by Regulation of Bcl-2 and Bax. Int J Gynecol Cancer. 2017;27:1596-601.

13. Forget MA, Desrosiers RR, Beliveau R. Physiological roles of matrix metalloproteinases: implications for tumor growth and metastasis. Can J Physiol Pharmacol. 1999;77:465-80.
14. Andreasen PA, Egelund $\mathrm{R}$, Petersen $\mathrm{HH}$. The plasminogen activation system in tumor growth, invasion, and metastasis. Cell Mol Life Sci. 2000;57:25-40.

15. Hildenbrand $\mathrm{R}$, Allgayer $\mathrm{H}$, Marx A, et al. Modulators of the urokinase-type plasminogen activation system for cancer. Expert Opin Investig Drugs. 2010;19:641-52.

16. Huang SF, Chu SC, Hsieh YH, et al. Viola Yedoensis Suppresses Cell Invasion by Targeting the Protease and NF-kappaB Activities in A549 and Lewis Lung Carcinoma Cells. Int J Med Sci. 2018;15:280-90.

17. Hung TW, Chen PN, Wu HC, et al. Kaempferol Inhibits the Invasion and Migration of Renal Cancer Cells through the Downregulation of AKT and FAK Pathways. Int J Med Sci. 2017;14:984-93.

18. Lin CY, Chen PN, Hsu LS, et al. Inhibition of the invasion and migration of renal carcinoma 7860 si3 cells in vitro and in vivo by Koelreuteria formosana extract. Mol Med Rep. 2014;10:3334-42.

19. Chu SC, Chiou HL, Chen PN, et al. Silibinin inhibits the invasion of human lung cancer cells via decreased productions of urokinase-plasminogen activator and matrix metalloproteinase-2. Mol Carcinog. 2004;40:143-9.

20. Hsieh YS, Chu SC, Yang SF, et al. Silibinin suppresses human osteosarcoma MG-63 cell invasion by inhibiting the ERK-dependent c-Jun/AP-1 induction of MMP-2. Carcinogenesis. 2007;28:977-87.

21. Yang SF, Chu SC, Liu SJ, et al. Antimetastatic activities of Selaginella tamariscina (Beauv.) on lung cancer cells in vitro and in vivo. J Ethnopharmacol. 2007;110:483-9.

22. Calautti E, Cabodi S, Stein PL, et al. Tyrosine phosphorylation and src family kinases control keratinocyte cell-cell adhesion. J Cell Biol. 1998;141:1449-65.

23. Wang WC, Tsai JJ, Kuo CY, et al. Non-proteolytic house dust mite allergen, Der $\mathrm{p} 2$, upregulated expression of tight junction molecule claudin-2 associated with Akt/GSK-3beta/beta-catenin signaling pathway. J Cell Biochem. 2011;112:1544-51.

24. Kim HJ, Kim YM, Lim S, et al. Ubiquitin C-terminal hydrolase-L1 is a key regulator of tumor cell invasion and metastasis. Oncogene. 2009:28:117-27.

25. Fuhrmann A, Banisadr A, Beri P, et al. Metastatic State of Cancer Cells May Be Indicated by Adhesion Strength. Biophys J. 2017;112:736-45.

26. Akhurst RJ, Derynck R. TGF-beta signaling in cancer--a double-edged sword. Trends Cell Biol. 2001;11:S44-51.

27. Lin $\mathrm{CH}$, Lin $\mathrm{HH}$, Kuo $\mathrm{CY}$, et al. Aeroallergen Der p 2 promotes motility of human non-small cell lung cancer cells via toll-like receptor-mediated up-regulation of urokinase-type plasminogen activator and integrin/focal adhesion kinase signaling. Oncotarget. 2017;8:11316-28.

28. Jin EJ, Park KS, Bang OS, et al. Akt signaling regulates actin organization via modulation of MMP-2 activity during chondrogenesis of chick wing limb bud mesenchymal cells. J Cell Biochem. 2007;102:252-61.

29. Rath N, Olson MF. Rho-associated kinases in tumorigenesis: re-considering ROCK inhibition for cancer therapy. EMBO Rep. 2012;13:900-8.

30. Kagawa Y, Matsumoto S, Kamioka Y, et al. Cell cycle-dependent Rho GTPase activity dynamically regulates cancer cell motility and invasion in vivo. PLoS One. 2013;8:e83629.

31. Li Z, Liu H, Zhong $\mathrm{Q}$, et al. LncRNA UCA1 is necessary for TGF-beta-induced epithelial-mesenchymal transition and stemness via acting as a ceRNA for Slug in glioma cells. FEBS Open Bio. 2018;8:1855-65.

32. Sun $\mathrm{Z}, \mathrm{Cao} \mathrm{B}, \mathrm{Wu}$ J. Protease-activated receptor 2 enhances renal cell carcinoma cell invasion and migration via PI3K/AKT signaling pathway. Exp Mol Pathol. 2015;98:382-9.

33. Duffy MJ. Urokinase-type plasminogen activator: a potent marker of metastatic potential in human cancers. Biochem Soc Trans. 2002;30:207-10. 